\title{
Hemolytic Uremic Syndrome: MR Findings of CNS Complications
}

Hemolytic uremic syndrome is a multisystem disorder characterized by the presence of microangiopathic hemolytic anemia, thrombocytopenia, and acute oliguric or anuric renal failure. It typically affects young children and adolescents, and most of the patients are less than 5 years old [1]. There is no sexual predilection. Neurologic complications include altered mental status (coma, stupor), personality changes, seizures, transient hemiparesis, and cortical blindness [2]. Numerous cases of the CNS complications of this syndrome have been evaluated by using CT [1-6], but few reports have mentioned the MR findings [7].

\section{Case Report}

A 21/2-year-old boy had had bloody diarrhea for 6 days. On physical examination, the child was irritable, pale, dehydrated, afebrile, and normotensive. The neurologic examination was remarkable only for the finding of lethargy. Multiple petechial hemorrhages were present on the child's buttocks and perineum. The WBC count was 48,000 / $\mu \mathrm{l}$, with a marked shift to the left; hemoglobin was $9.9 \mathrm{~g} / \mathrm{dl}(99 \mathrm{~g} / \mathrm{l})$; and hematocrit was $30.3 \%(0.30)$. Serum levels were sodium, 122 $\mathrm{mEq} / \mathrm{l}(122 \mathrm{mmol} / \mathrm{l}) ;$ potassium, $4.9 \mathrm{mEq} / \mathrm{l}(4.9 \mathrm{mmol} / \mathrm{l})$; blood urea nitrogen, $83 \mathrm{mg} / \mathrm{dl}$ (29.6 mmol urea/l); and creatinine, $3.6 \mathrm{mg} / \mathrm{dl}$ (318 $\mu \mathrm{mol} / \mathrm{l})$. The diagnosis was hemolytic uremic syndrome and possible sepsis.

The patient became progressively oliguric and hypertensive, requiring hemodialysis. His hematocrit dropped to $19.7 \%(0.20)$, and he was transfused with $100 \mathrm{ml}$ of packed RBCs, after which he became more alert. He continued to be oliguric and hypertensive, and 8 days after admission, he became unresponsive, with gasping respiration and then generalized tonic-clonic seizures. CT at this time was normal. The patient was intubated and received peritoneal dialysis and anticonvulsant therapy, after which he became slightly more responsive. An electroencephalogram showed intermittent epileptiform activity without associated motor seizures. The renal and neurologic status gradually stabilized, and the patient was extubated 15 days after admission. MR obtained 22 days after admission (Fig. 1) showed bilateral lesions in the basal ganglia that had increased signal intensity on T1- and T2-weighted images and a large right posterior parietooccipital nonhemorrhagic infarction. Severe neurologic impairment persisted 1 month after admission.

\section{Discussion}

Early dialysis and improved supportive care have reduced the mortality in hemolytic uremic syndrome from approximately $21 \%$ in 1974 to $4-7 \%$ in $1985[8,9]$. Neurologic complications still affect 20$50 \%$ of patients and indicate a worse prognosis, with increased mortality and morbidity [2, 9]. This may be explained partially by metabolic derangement, including renal failure and hypertension. The renal pathologic changes have been well documented and are characterized by multiple microthrombi and intravascular coagulation. The neuropathologic findings have been reported in several series. Upadhyaya et al. [5] found multiple microthrombi and microangiopathy in two of three brains studied. Other series have failed to confirm these findings. Rooney et al. [6] found nonspecific hypoxic changes and cerebral edema in seven patients. Gianantonio et al. [1] reviewed autopsies of 47 patients who had hemolytic uremic syndrome and found cerebral edema, gross and microscopic parenchymal hemorrhage, and fibrin deposits in the choroid plexus, but no cerebral microthrombi. Trevathan and Dooling [4] reported three cases (one autopsy proved) of large-vessel thrombotic infarctions without evidence of small-vessel disease. Hahn et al. [2] studied three patients and found cerebral edema and hemorrhagic infarctions in the basal ganglia, thalamus, hippocampus, and cortex. No microthrombi or large-vessel thrombi were identified. In summary, the spectrum of documented neuropathologic changes in the syndrome includes hemorrhagic or bland infarction in both large and small vessels, hypoxic changes, and cerebral edema.

In our case, CT performed $12 \mathrm{hr}$ after the onset of seizures was normal. MR done 14 days later showed increased signal intensity throughout the basal ganglia on T1-, proton-density-, and T2weighted images (Fig. 1). These findings are consistent with subacute hemorrhagic infarction. Only one other case with MR findings has been reported. DiMario et al. [7] found punctate foci of increased signal intensity in the caudate, putamen, and globus pallidus, which they ascribed to subacute hemorrhagic infarction. In addition to subacute hemorrhagic infarction, a number of other processes can cause hyperintense signal in the basal ganglia, including microvascular angiopathy without hemorrhage, perivascular and interstitial mineral deposition, gliosis, neurofibromatosis [10], and tuberous sclerosis [11]. Although the finding of increased signal intensity in the basal ganglia in our case was nonspecific, subacute hemorrhagic infarction correlated best with the clinical history of an acute neurologic event 14 days before the MR study. CT was not obtained at the time of the MR, and we have no histologic correlation for this case, so hemorrhagic infarction could not be confirmed absolutely. The large lesion in the posterior right parietal and occipital lobes, which was isointense on T1-weighted images and hyperintense on proton-density- and T2-weighted images, is most consistent with a nonhemorrhagic infarction. Both hemorrhagic and nonhemorrhagic infarctions have been reported in autopsy series of patients who had

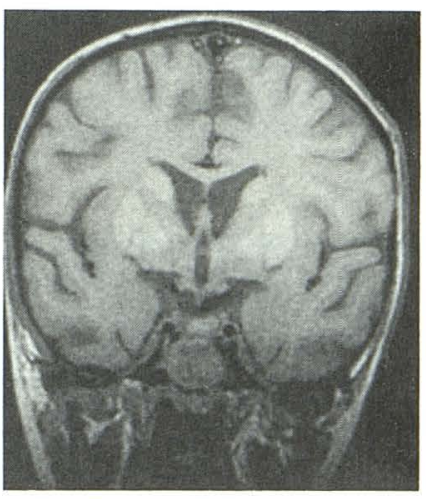

A

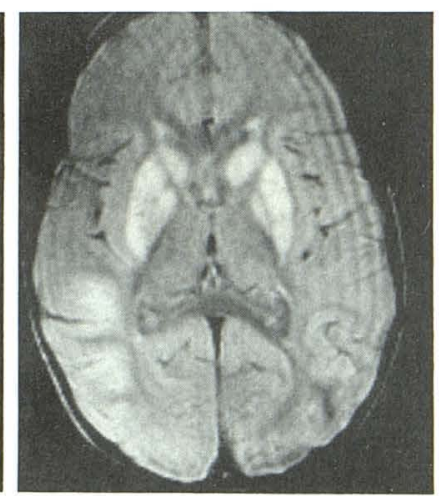

B
Fig. 1.-MR findings in CNS complications of hemolytic uremic syndrome.

$A$, Coronal image, $600 / 20 / 2$, shows increased signal intensity in caudate, putamen, and globus pallidus bilaterally.

$B$, Axial image, $2000 / 40$, shows increased signal intensity in both basal ganglia and in right posterior parietooccipital area. 
hemolytic uremic syndrome. The MR findings in our case indicate that the patient had both hemorrhagic and nonhemorrhagic infarction. James W. Sherwood William A. Wagle Albany Medical Center Albany, NY 12208

\section{REFERENCES}

1. Gianantonio CA, Vitaccio M, Mendilaharzu F, Gallo GE, Sojo ET. The hemolytic uremic syndrome. Nephron 1973;11:174-192

2. Hahn JS, Havens PL, Higgins JJ, O'Rouke PP, Estroff JA, Strand R. Neurologic complications of hemolytic uremic syndrome. J Child Neurol 1989;4:108-113

3. Crisp DE, Siegler RL, Bale JF, Thompson JA. Hemorrhagic cerebral infarction in the hemolytic uremic syndrome. $J$ Pediatr 1981;99:273-276

4. Trevathan E, Dooling EC. Large thrombotic stroke in hemolytic uremic syndrome. J Pediatr 1987;111:863-866

5. Upadhyaya K, Barwick K, Fishaut M, Kashgarian M, Siegel NJ. The importance of nonrenal involvement in the hemolytic uremic syndrome. Pediatrics 1980;65:115-120

6. Rooney JC, Anderson RM, Hopkins IJ. Clinical and pathological aspects of central nervous system involvement in the hemolytic uremic syndrome. Aust Pediatr 1971;7:28-33

7. DiMario FJ, Brone-Stewart H, Sherbotie J, Turner ME. Lacunar infarction of the basal ganglia as a complication of hemolytic uremic syndrome. Clin Pediatr 1987;26:586-590

8. Proesmans W, Eeckels R. Has heparin changed the prognosis in the hemolytic uremic syndrome? Clin Nephrol 1974;2:169-173

9. Sheth KJ, Swick HM, Haworth N. Neurological involvement in the hemolytic uremic syndrome. Ann Neurol 1986;19:90-93

10. Aoki S, Barkovich AJ, Nishimura K, et al. Neurofibromatosis types 1 and 2: cranial MR findings. Radiology 1989;172:527-534

11. Nixon JR, Houser OW, Gomez MR, Okazaki H. Cerebral tuberous sclerosis: MR imaging. Radiology 1989;170:869-873 